\title{
La importancia de la contabilidad financiera en los grados de ADE de las universidades españolas según los créditos impartidos
}

\author{
The importance of financial accounting in Business Administration \\ and Management degrees at the Spanish universities
}

\author{
Julián Martínez Vargas (julian.martinez@uv.es) \\ Departamento de Contabilidad, Universidad de Valencia, España \\ http://dx.doi.org/10.12795/EDUCADE.2017.i08.06
}

\begin{abstract}
RESUMEN: Este trabajo tiene como objetivo analizar si en el grado más ofertado por las universidades españolas como es el de Administración y Dirección de Empresas, puede haber diferencias relevantes de contenido, centrándonos en una de las áreas que forma parte de su columna vertebral como es la contabilidad y, más concretamente, la contabilidad financiera.
\end{abstract}

\begin{abstract}
Valoramos los créditos impartidos en todas la universidades, tanto públicas como privadas, comprobando que hay diferencias significativas y destacando aquellas en las que la materia adquiere una notable relevancia acorde con la importancia que tradicionalmente ha tenido en estos estudios (Sevilla, Almería, Granada, Pablo de Olavide, Las Palmas, León, Europea de Madrid, UNED, Navarra, Alicante, Extremadura, La Coruña, Islas Baleares, La Rioja, Miguel Hernández, Zaragoza, Cantabria, País Vasco, UDIMA, Murcia y Santiago de Compostela), frente a las que han reducido su presencia a la mínima expresión (Carlos III, IE Universidad, Autónoma de Barcelona, Valencia y Oviedo), lo que puede suponer una formación muy dispar dependiendo de la localización de la universidad.
\end{abstract}

También se pone de manifiesto que las universidades que han ofertado de forma paralela el nuevo grado de Finanzas y Contabilidad han perdido peso en contabilidad financiera pero no de forma relevante.

PALABRAS CLAVE: Redes sociales, Plataforma virtual, Innovación docente, hasta cinco.

\begin{abstract}
This study aims to analyse if in the most offered degree by Spanish universities, that is Business Administration and Management, there may be significant differences in content. We focus on one area that is part of its vertebral column such as the accounting and, more specifically, financial accounting.

We make a valuation of taught credits in the state and private universities, observing that there are significant differences. We highlight those in which the subject acquires a remarkable relevance in agreement with the importance that it has traditionally had in these studies (Sevilla, Almería, Granada, Pablo de Olavide, Las Palmas, León, Europea de Madrid, UNED, Navarra, Alicante, Extremadura, La Coruña, Islas Baleares, La Rioja, Miguel Hernández, Zaragoza, Cantabria, País Vasco, UDIMA, Murcia y Santiago de Compostela), versus those that have reduced their presence to the bare minimum (Carlos III, IE Universidad, Autónoma de Barcelona, Valencia y Oviedo). This situation might cause a very different formation depending on the location of universities.

This paper also shows that the universities that offer the new Finance and Accounting degree at the same time that ADE degree have influenced negatively in the financial accounting but not in a relevant way.
\end{abstract}

Artículo. Recibido: 01-03-17 - Versión revisada: 14-07-17, 02-11-17 Aceptado: 15-11-17

Licencia Creative Commons BY NC ND · $2017 \cdot$ Asociación Española de Contabilidad y Administración de Empresas - AECA 
KEYWORDS: Financial accounting; Degree; Business Administration and Management; Education; University

\section{INTRODUCCIÓN}

La última reforma de los estudios universitarios para su adaptación al sistema de grados responde al proceso de convergencia con el resto de los países europeos, surgido a partir del compromiso adquirido en la Declaración de Bolonia. Pero para que podamos hablar de una verdadera convergencia, primero tenemos que lograrlo en nuestro propio país, ya que no alcanzaríamos dicho objetivo si un mismo grado difiere notablemente en los contenidos abordados, dependiendo de la Comunidad Autónoma o de la Universidad en la que se imparta.

Según el artículo 9 del Real Decreto 1393/2007, por el que se establece la ordenación de las enseñanzas universitarias oficiales, "las enseñanzas de Grado tienen como finalidad la obtención por parte del estudiante de una formación general, en una o varias disciplinas, orientada a la preparación para el ejercicio de actividades de carácter profesional" y una de esas disciplinas debería ser la contabilidad financiera, ya que resulta imprescindible para el desarrollo de la mayoría de las actividades relacionadas con la gestión empresarial.

Por su lado, el artículo 10 de ese mismo Real Decreto establece que "las enseñanzas de Master tienen como finalidad la adquisición por el estudiante de una formación avanzada, de carácter especializado o multidisciplinar, orientada a la especialización académica o profesional, o bien a promover la iniciación en tareas investigadoras". La contabilidad financiera también se concibe como la base fundamental de cualquier posgrado que aborde la auditoría de cuentas como especialización, siendo a su vez parte importante para la formación avanzada en materias relacionadas con el análisis, las finanzas y la tributación empresarial.

Centrándonos en los grados, el de Administración y Dirección de Empresas (ADE) es, con creces, el más ofertado por las universidades españolas, lo que se debe explicar lógicamente por su elevada demanda. La práctica totalidad de universidades españolas, tanto públicas como privadas, ofertan este grado y su éxito no es ajeno a las posibilidades laborales que ofrece y a las buenas perspectivas de trabajo (Informe Infoempleo de Adecco, 2016 y III Ranking Universidad-Empresa de la Fundación Everis, 2017).

Según el estudio de Everis (2017), existe una elevada concentración de la contratación en determinados ámbitos de conocimiento e incluso en titulaciones concretas. Por tercer año consecutivo, las dos titulaciones con un mayor nivel de contratación son ADE e Ingeniería Industrial, aglutinando entre las dos una cuarta parte de las contrataciones. En otro documento publicado por la Federación de Expertos Contables Europea (FEE, 2015) se destaca la responsabilidad de los profesionales de la contabilidad en el desarrollo y correcto funcionamiento de los sistemas fiscales de los estados europeos.

Sin embargo, los contenidos abordados en los grados de ADE pueden variar mucho de una universidad a otra y una prueba de ello son las diferencias que encontramos en una de sus áreas más importantes como es la contabilidad. Y esta circunstancia no se corresponde con lo establecido en el Real Decreto 1393/2007 donde se pretende, entre otras cosas, "potenciar la apertura hacia los estudiantes procedentes de otros países del Espacio Europeo de Educación Superior y de otras áreas geográficas, marcando una nueva estrategia en el contexto global de la Educación Superior", así como "fomentar la movilidad de los estudiantes, tanto dentro de Europa, como con 
otras partes del mundo, y sobre todo la movilidad entre las distintas universidades españolas". Pero si esta globalización no se alcanza en España, difícilmente lo habremos logrado con Europa.

Partiendo de estas premisas y teniendo en cuenta que, como veremos a lo largo de este trabajo, hay diferencias importantes entre los grados de ADE de la universidades españolas en lo que a la contabilidad financiera se refiere, pretendemos que en el contexto actual en el que se avecinan nuevas reformas en los grados y másteres, se aproveche para tratar de recuperar el terreno perdido por la contabilidad en aquellas universidades donde no se le ha dado la importancia que tradicionalmente ha tenido.

Así, empezaremos por ver cómo ha ido cambiando en los últimos años la asignación de créditos a los estudios superiores de Administración y Dirección de Empresas y consecuentemente a las diferentes áreas de conocimiento, para llegar al contexto actual de los grados, con una justificación general de clara orientación laboral, cuya concreción se basa en la propuesta realizada en el Libro Blanco del Título de Grado de Economía y Empresa.

Con los datos de los planes de estudio de todos los grados de ADE contrastaremos de manera efectiva las diferencias en créditos de contabilidad financiera entre los distintos centros universitarios, valorando si son estadísticamente significativas, si dependen de que el centro sea público o privado o si puede deberse al hecho de que la universidad ofrezca de forma paralela el Grado de Finanzas y Contabilidad. También hacemos referencia a cómo puede estar influyendo en la docencia de la contabilidad la investigación y el actual sistema de evaluación y reconocimiento de méritos que, según han apuntado ya diferentes autores, está provocando un distanciamiento con la práctica contable.

\section{DE LAS LICENCIATURAS A LOS GRADOS}

Con el Real Decreto 1393/2007 se estableció que las universidades debían reformar sus estudios superiores para el curso 2010/2011, estructurando las titulaciones superiores en grados y posgrados. En esta línea, la Ley Orgánica de Universidades (LOU), en sU artículo 87 De la integración en el espacio Europeo de enseñanza superior, se indica que "en el ámbito de sus respectivas competencias el gobierno, las comunidades autónomas y las universidades, adoptarán las medidas necesarias para la plena integración del sistema español en el espacio europeo de enseñanza superior". Y en este proceso de integración, el Ministerio de Educación, Cultura y Deporte, mediante un documento marco de 2003, ya proponía un primer nivel de grado que permitiera obtener un título con cualificación profesional en el mercado laboral europeo. Por lo tanto, la orientación de los grados es profesional, estableciéndose que debe proporcionar una "formación en la que se integren las competencias genéricas básicas, las competencias transversales relacionadas con la formación integral de las personas y las competencias más específicas que posibiliten una orientación profesional". En un segundo nivel y una vez superado el grado se podría acceder al postgrado, más orientado a la especialización a través de un máster y/o el doctorado.

A partir de estas consideraciones debemos valorar y reflexionar sobre si la contabilidad financiera debe tener un papel relevante en esta orientación laboral de los nuevos grados $y$, por lo tanto, mantener una presencia en créditos acorde con la que tradicionalmente ha tenido en estas enseñanzas.

Según el estudio realizado por Cavero y Reig (2013), en la antigua Licenciatura en Ciencias Empresariales, los créditos obligatorios de contabilidad representaban una media de 45,20 lo que supone un peso del $15,71 \%$ del total de créditos $(287,70)$. En la Licenciatura en ADE, la media de créditos es de 35,46 y del $15,68 \%$ del total $(226,08)$. 
En el grado de ADE, para determinar los créditos de las diferentes asignaturas se tomó como referencia lo establecido en el Libro Blanco del Título de Grado de Economía y Empresa elaborado por la Conferencia de Decanos de Economía y Empresa (CONFEDE, 2004). Así, se propone ofertar 24 créditos de contabilidad de los 160 de formación básica y obligatoria, lo que representaría un 15\%, lo que según el citado estudio de Cavero y Reig se concretó en una media de 27,91 créditos obligatorios de contabilidad, es decir, un $14.74 \%$ de los 189, 24 créditos de formación básica y obligatoria de la media de las universidades públicas españolas. Por lo tanto, en torno a ese $15 \%$ propuesto por el Libro Blanco. Sin embargo, resulta interesante ver cómo se llega a esa propuesta de créditos en contabilidad del Libro Blanco.

\subsection{LA PROPUESTA DE CRÉDITOS EN CONTABILIDAD DEL LIBRO BLANCO}

Dado que los grados tienen un claro objetivo de cualificación profesional, en el citado Libro Blanco se incorpora como anexo E un trabajo donde se realiza un análisis de los principales puestos de trabajo que pueden desarrollar los licenciados en ADE, definiendo sus funciones (financiero-contable, comercial, organización y global), las responsabilidades que debe asumir y las habilidades exigidas para el desempeño de cada puesto de trabajo. Para la selección de los puestos de trabajo demandados y de las cualidades exigidas a los candidatos se utiliza básicamente una muestra de anuncios de prensa general y de prensa financiera de ámbito nacional.

Con los datos relativos a demanda de empleo y formación se estudia el grado de adecuación de los estudios en ADE para el desarrollo de cada puesto de trabajo, solicitando la opinión de personal académico y de licenciados con experiencia profesional y valorando la utilidad de las materias contenidas en los planes de estudio españoles para el desarrollo de los puestos de trabajo definidos. Englobadas las materias en las áreas de conocimiento de contabilidad, derecho, economía, finanzas, historia, idiomas, informática, marketing, matemáticas, organización y sociología, la más valorada por los profesionales es contabilidad con un $14,27 \%$, seguida de organización con un $13,05 \%$ y de finanzas con un $12,98 \%$. Sin embargo, cuando estas valoraciones se promedian con la de los académicos, que a las materias del área de organización otorgan una ponderación del 22,81\% (frente al $13,05 \%$ de los profesionales), del $17,23 \%$ al marketing (frente a un $10,29 \%$ de los profesionales) y del 12,54\% a la contabilidad, hacen que el área de organización se sitúe en primer lugar con una ponderación del $17,93 \%$, el marketing en segundo lugar (según los profesionales es el sexto) con un $13,76 \%$ y la contabilidad descienda hasta el tercer lugar con un $13,41 \%$. De esta forma, en las valoraciones finales la contabilidad pierde un punto porcentual con respecto a la opinión de los profesionales, organización gana casi 5 puntos y el marketing 3,5 puntos.

Pero, ¿no partíamos de una base según la cual lo grados tendrían una orientación profesional? ¿̇cómo puede ser que la opinión de los académicos, muchos de los cuales carecen de experiencia profesional, se valore con el mismo peso que la de los profesionales haciendo que cambien tanto las valoraciones finales con respecto a la opinión de los profesionales?

Así, según las conclusiones de este estudio, el plan de estudios pivotaría sobre cuatro pilares fundamentales: la organización del empresas (con un 17,93\% y 43,02 créditos), el marketing (con un $13,76 \%$ y 33,02 créditos), la contabilidad (con un 13,41\% y 32, 17 créditos) y las finanzas (con un $11,95 \%$ y 28,68 créditos).

Finalmente, el Libro Blanco, teniendo en cuenta estos datos y tras examinar las experiencias de los estudios de economía y empresa de las universidades de 9 países europeos que ya habían hecho la adaptación al EEES, para un grado en ADE de 4 
años y 240 créditos como el que se pone en marcha en España, propone ofertar 24 créditos de contabilidad de los 160 de formación básica y obligatoria, lo que representa un 15\%, por debajo en todo caso de los 26 créditos y el 16,25\% del bloque temático correspondiente a organización de empresas.

Por lo tanto, teniendo en cuenta el claro objetivo de cualificación profesional de los grados y la importancia dada por los profesionales a la contabilidad, su peso específico en el grado de ADE debería ser de los más importantes y, como veremos a continuación, no es lo que ocurre en muchas de nuestras universidades.

En este sentido, tratando de reducir la brecha entre formación y empleo, los empresarios españoles a través de su Libro Blanco "La educación importa" (CEOE, 2017) establecen que es necesario mejorar los mecanismos de transición de la educación al empleo y la intensificación de los vínculos recíprocos entre educación y empresa. Para ello proponen una serie de actuaciones en la formación universitaria tales como incorporar a las organizaciones empresariales en el Consejo de Universidades e implicarlas en la gobernanza de las instituciones universitarias orientadas hacia el sistema productivo, o incorporar al mundo empresarial de un modo eficaz en la definición de los currículos de Grado y de Postgrado y en su actualización, a fin de asegurar las competencias profesionales que los graduados deberán desarrollar en los entornos de desempeño laboral.

\section{METODOLOGÍA}

Para ver la importancia de la contabilidad en los grados de ADE ofertados por las universidades españolas hemos consultado sus planes de estudio y las guías docentes en las páginas web, centrándonos en los créditos dedicados a esta materia. Con los datos obtenidos analizamos si hay diferencias estadísticamente significativas entre universidades públicas y privadas o entre las universidades que han incorporado como nuevo grado el de Finanzas y Contabilidad, por lo que podrían haber reducido la presencia de la contabilidad en ADE en beneficio de aquél. También veremos que hay universidades en las que la contabilidad se ha reducido drásticamente a partir de la última reforma de los grados, estando muy por debajo de la media y de lo que se considera normal en la mayoría de universidades y en los contenidos que tradicionalmente se ha exigido en la formación en ADE.

En el estudio no vamos a considerar los créditos de asignaturas de contabilidad de costes ya que, a diferencia de lo que ocurre con las asignaturas de contabilidad financiera, la discrecionalidad entre las diferentes universidades es mínima, dedicándose por lo general entorno a 6 créditos del grado. Para elevar al total los créditos en contabilidad bastaría con sumar esos 6-7 créditos a los de contabilidad financiera para obtener una buena aproximación.

Por lo tanto, nos hemos centrado en las asignaturas de contabilidad financiera por apreciar que su oferta en créditos es muy diferente dependiendo de la Universidad y porque constituyen la base necesaria para una especialización en cuestiones tales como la administración de empresas, la auditoría y la fiscalidad empresarial. En este sentido, consideramos que la formación obligatoria en contabilidad financiera es primordial y debe abordarse con un mínimo de créditos adecuado antes de afrontar otras materias relacionadas. En primer lugar nos hemos fijado en los créditos obligatorios de asignaturas con contenidos de contabilidad financiera (introducción a la contabilidad, contabilidad financiera I, contabilidad financiera II, contabilidad avanzada, etc.) para, a continuación, valorar los créditos obligatorios relacionados con la contabilidad financiera (análisis de estados contables en la mayoría de universidades y en menor proporción auditoría y consolidación contable, que también 
se suelen ofrecer como materias optativas), cuyo buen desarrollo y comprensión requiere una formación adecuada en contabilidad financiera (Figura 1).

Consideramos que una baja dedicación a la contabilidad financiera en su formación básica no se puede compensar con una amplia oferta de materias optativas para cuya elección, buena aceptación y desarrollo adecuado se hace imprescindible haber dedicado el tiempo mínimo necesario a la contabilidad financiera.

\section{RESULTADOS}

Para realizar nuestro análisis hemos consultado los planes de estudio de todos los grados de ADE ofertados en España, lo que supone un total de 75, de los cuales 46 son de universidades públicas y 29 son de universidades privadas/católicas.

Figura 1. Distribución de las asignaturas de Contabilidad Financiera

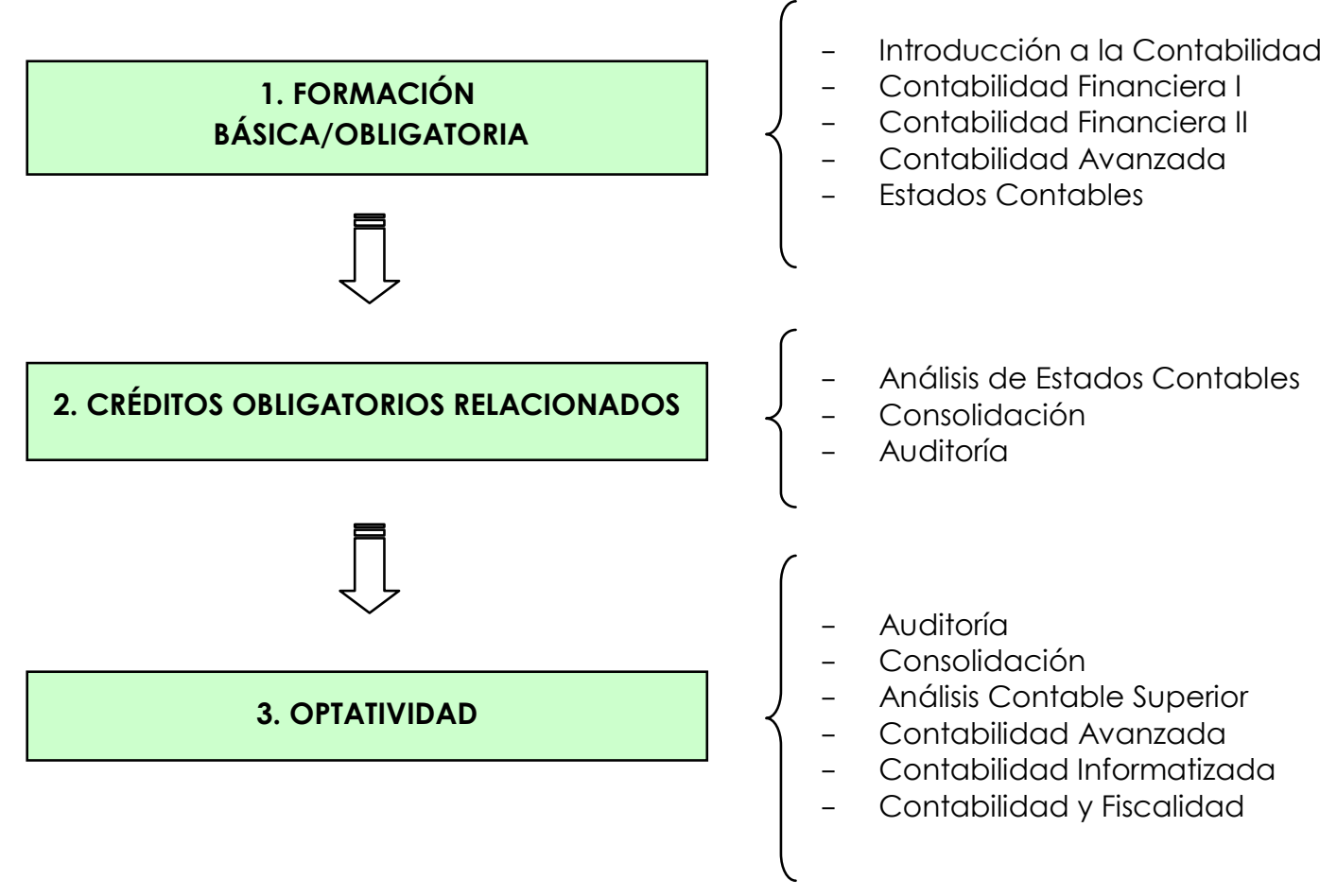

Si empezamos fijándonos en la frecuencia de los créditos obligatorios de contabilidad financiera por universidades (Tabla 1), podemos observar que lo más habitual es una asignación de 12 créditos (un $40 \%$ de las universidades), aunque también destaca la asignación de 18 créditos (un 25,3\%). Pero lo más destacado sería establecer que el $80 \%$ de los grados de ADE asigna a esta materia al menos 12 créditos. 
Tabla 1. Frecuencia créditos obligatorios contab. fra.

\begin{tabular}{|c|r|r|r|}
\hline Créditos & Frecuencia & Porcentaje & Porcentaje acum. \\
\hline 6,0 & 8 & 10,7 & 10,7 \\
8,0 & 1 & 1,3 & 12,0 \\
9,0 & 3 & 4,0 & 16,0 \\
10,0 & 1 & 1,3 & 17,3 \\
10,5 & 1 & 1,3 & 18,7 \\
11,0 & 1 & 1,3 & 20,0 \\
12,0 & 30 & 40,0 & 60,0 \\
13,5 & 1 & 1,3 & 61,3 \\
15,0 & 5 & 6,7 & 68,0 \\
15,6 & 1 & 1,3 & 69,3 \\
16,0 & 1 & 1,3 & 70,7 \\
16,5 & 1 & 1,3 & 72,0 \\
18,0 & 19 & 25,3 & 97,3 \\
19,5 & 1 & 1,3 & 98,7 \\
21,0 & 1 & 1,3 & 100,0 \\
Total & 75 & 100,0 & \\
\hline
\end{tabular}

De forma que, tal y como observamos en la Tabla 2, la media de créditos obligatorios de contabilidad financiera de las universidades españolas está en 13,25 , a los que si añadimos otros 5,96 créditos de asignaturas obligatorias relacionadas con la contabilidad financiera nos da un total de 19,21 créditos. Pero también debemos destacar la desviación estándar de más de 5 créditos y que los créditos obligatorios dedicados a la contabilidad financiera oscilan entre 6 y 21 , mientras que los créditos totales están entre 6 y 36.

Tabla 2. Créditos obligatorios de contabilidad financiera

\begin{tabular}{|l|l|l|l|l|l|}
\hline & N & Mínimo & Máximo & Media & Desv. estándar \\
\hline Créd. Oblig. CF & 75 & 6,0 & 21,0 & 13,25 & 3,92 \\
Créd. Oblig. Relac. CF & 75 & 0 & 18,0 & 5,96 & 3,37 \\
TOTAL CRÉD. OBLIG. & 75 & 6,0 & 36,0 & 19,21 & $\mathbf{5 , 2 7}$ \\
\hline
\end{tabular}

Si hacemos una diferenciación entre las universidades públicas y privadas (Tabla 3) empezamos a observar las primeras diferencias importantes, ya que en las universidades públicas se dedica de media algo más de 2 créditos adicionales a las asignaturas obligatorias de contabilidad financiera y algo más de medio crédito también a las asignaturas relacionadas con la contabilidad financiera. 
Tabla 3. Créditos obligatorios de contabilidad financiera según Púb./Priv.

\begin{tabular}{|l|l|l|l|l|}
\hline \multicolumn{2}{|l|}{ Pública/Privada } & Créd. Oblig. CF & Créd.Oblig. relac. CF & TOTAL OBLIG. \\
\hline Pública & Media & 14,08 & 6,19 & 20,27 \\
$N=46$ & Desviación estándar & 4,10 & 2,87 & 5,17 \\
\hline Privada & Media & 11,93 & 5,59 & 17,52 \\
$N=29$ & Desviación estándar & 3,27 & 4,07 & 5,06 \\
\hline Total & Media & $\mathbf{1 3 , 2 5}$ & 5,96 & 19,21 \\
$N=75$ & Desviación estándar & 3,92 & 3,37 & 5,27 \\
\hline
\end{tabular}

Estos resultados vienen a coincidir con los del estudio de Cavero y Reig (2013) para las universidades públicas, ya que ellos obtuvieron una media de créditos obligatorios de contabilidad de 27,91. Si a los 20,27 que se obtienen en este estudio para la contabilidad financiera, sumamos los créditos dedicados a la contabilidad de costes que son los únicos que faltan y que están entorno a los 6-7 créditos, llegamos a unas valoraciones similares. Pero lo que verdaderamente nos preocupa y queremos poner de manifiesto en este trabajo es el hecho de que haya diferencias importantes entre las universidades, para lo que debemos tomar en consideración el dato de la desviación estándar superior a los 5 créditos.

Para valorar si esa diferencia total de 2,75 créditos entre las universidades públicas y las privadas se puede considerar estadísticamente significativa, utilizaremos la prueba paramétrica † de Student que permite contrastar hipótesis de igualdad de medias entre dos variables o poblaciones (Tabla 4). Rechazando la hipótesis de igualdad de varianzas según la prueba de Levene y dado que el coeficiente de significación bilateral es inferior a 0,05, podemos establecer que los créditos obligatorios de contabilidad financiera son significativamente diferentes entre las universidades públicas y las privadas, teniendo más peso en las universidades públicas.

Tabla 4. Prueba de muestras independientes para créd. obligat de contab. fra. según priv/públ

\begin{tabular}{|c|c|c|c|c|c|c|c|c|c|}
\hline & \multicolumn{2}{|c|}{$\begin{array}{c}\text { Prueba Levene } \\
\text { calidad } \\
\text { varianzas }\end{array}$} & \multicolumn{7}{|c|}{ prueba t para la igualdad de medias } \\
\hline & \multirow[b]{2}{*}{$\mathrm{F}$} & \multirow[b]{2}{*}{ Sig. } & \multirow[b]{2}{*}{$t$} & \multirow[b]{2}{*}{$\mathrm{gl}$} & \multirow{2}{*}{$\begin{array}{l}\text { Sig. } \\
\text { (bilat.) }\end{array}$} & \multirow{2}{*}{$\begin{array}{l}\text { Difer. de } \\
\text { medias }\end{array}$} & \multirow{2}{*}{$\begin{array}{l}\text { Difer. } \\
\text { error } \\
\text { están } \\
\text { d. }\end{array}$} & \multicolumn{2}{|c|}{$\begin{array}{c}\text { 95\% de interv. } \\
\text { de confianza } \\
\text { diferen. }\end{array}$} \\
\hline & & & & & & & & Inferior & Super. \\
\hline $\begin{array}{l}\text { Se asumen } \\
\text { varianzas iguales } \\
\text { No se asumen } \\
\text { varianzas iguales }\end{array}$ & 6,943 & 0,010 & $\begin{array}{l}-2,373 \\
-2,497\end{array}$ & $\begin{array}{r}73 \\
68,881\end{array}$ & $\begin{array}{l}0,020 \\
\mathbf{0 , 0 1 5}\end{array}$ & $\begin{array}{l}-2,1416 \\
-2,1416\end{array}$ & $\begin{array}{l}0,9025 \\
\mathbf{0 , 8 5 7 6}\end{array}$ & $\begin{array}{l}0,3429 \\
\mathbf{0 , 4 3 0 7}\end{array}$ & $\begin{array}{l}3,9403 \\
3,8525\end{array}$ \\
\hline
\end{tabular}

Nota: La equivalente no paramétrica $U$ de Mann-Whitney arroja un resultado similar (sig $0,016)$. 
Si de forma complementaria consideramos como variable dependiente los créditos obligatorios totales de contabilidad financiera, en la Tabla 5 podemos comprobar que al considerar todos los créditos de contabilidad financiera se siguen dando diferencias significativas entre las universidades públicas y privadas, siendo en este caso la diferencia de medias de casi 3 créditos, a la vez que la desviación estándar también es mayor en ambos tipos de universidades.

Uno de los factores a los que se ha apuntado como causa de una posible reducción de los créditos dedicados a la contabilidad en el grado de ADE es que en la misma universidad se imparta también un grado de Finanzas y Contabilidad (GFYC). Según Escobar y Jiménez (2009) el GFYC podría condicionar las asignaturas y créditos de contabilidad del grado de ADE. Tratando de verificar si efectivamente ésta podría ser una de las causas de las diferencias entre universidades, hemos hecho una clasificación entre las que ofertan este grado y las que no, cuyos resultados los podemos ver en la Tabla 6.

Tabla 5. Prueba de muestras independientes para TOTAL OBLIG. según privada/pública

\begin{tabular}{|c|c|c|c|c|c|c|c|c|c|}
\hline & \multicolumn{2}{|c|}{$\begin{array}{c}\text { Prueba Levene } \\
\text { calidad } \\
\text { varianzas }\end{array}$} & \multicolumn{7}{|c|}{ prueba t para la igualdad de medias } \\
\hline & \multirow[b]{2}{*}{$\mathrm{F}$} & \multirow[b]{2}{*}{ Sig. } & \multirow[b]{2}{*}{$t$} & \multirow[b]{2}{*}{ gl } & \multirow{2}{*}{$\begin{array}{c}\text { Sig. } \\
\text { (bilat.) }\end{array}$} & \multirow{2}{*}{$\begin{array}{l}\text { Difer. de } \\
\text { medias }\end{array}$} & \multirow{2}{*}{$\begin{array}{l}\text { Difer. } \\
\text { error } \\
\text { están } \\
\text { d. }\end{array}$} & \multicolumn{2}{|c|}{$\begin{array}{c}\text { 95\% de interv. } \\
\text { de confianza } \\
\text { diferen. }\end{array}$} \\
\hline & & & & & & & & Inferior & Super. \\
\hline $\begin{array}{l}\text { Se asumen } \\
\text { varianzas iguales } \\
\text { No se asumen } \\
\text { varianzas iguales }\end{array}$ & 0,924 & 0,340 & $\begin{array}{l}2,260 \\
2,272\end{array}$ & $\begin{array}{r}73 \\
60,680\end{array}$ & $\begin{array}{l}\mathbf{0 , 0 2 7} \\
0,027\end{array}$ & $\begin{array}{l}2,7489 \\
2,7489\end{array}$ & $\begin{array}{l}1,2163 \\
1,2101\end{array}$ & $\begin{array}{l}\mathbf{0 , 3 2 4 8} \\
0,3290\end{array}$ & $\begin{array}{l}5,1730 \\
5,1688\end{array}$ \\
\hline
\end{tabular}

Nota: La equivalente no paramétrica U de Mann-Whitney arroja un resultado similar (sig. 0,004).

La carga docente obligatoria de contabilidad financiera es inferior (aprox. 1.5 créditos) en las universidades donde también se oferta el GFYC. Sin embargo, la dispersión es también bastante alta en estas universidades, por lo que esta diferencia no llega a ser significativa. La tabla 7 muestra los resultados obtenidos en la prueba paramétrica para contrastar la igualdad de medias. Asumiendo la hipótesis de igualdad de varianzas y dado que el coeficiente de significación bilateral es superior a 0,05 , se acepta la hipótesis de igualdad de medias. Así, no podemos establecer que ésta sea una causa determinante.

Tabla 6. Créditos obligatorios de contabilidad financiera según GFYC NO/SI

\begin{tabular}{|c|l|r|r|r|}
\hline \multicolumn{2}{|c|}{ GFYC NO/SI } & Créd. Oblig. CF & Créd. Oblig. relac. CF & TOTAL OBLIG. \\
\hline No & Media & 13,48 & 6,14 & 19,62 \\
$N=53$ & Desviación estándar & 3,63 & 3,53 & 5,06 \\
\hline Sí & Media & 12,68 & 5,52 & 18,21 \\
$N=22$ & Desviación estándar & 4,60 & 2,98 & 5,75 \\
\hline Total & Media & $\mathbf{1 3 , 2 5}$ & 3,96 & $\mathbf{1 9 , 2 1}$ \\
$\mathrm{N}=75$ & Desviación estándar & 3,92 & 5,27 \\
\hline
\end{tabular}


Tabla 7. Prueba de muestras independientes créditos oblig. contab. fra. según GFYC Sí/NO

\begin{tabular}{|c|c|c|c|c|c|c|c|c|c|}
\hline & \multicolumn{2}{|c|}{$\begin{array}{c}\text { Prueba Levene } \\
\text { calidad } \\
\text { varianzas }\end{array}$} & \multicolumn{7}{|c|}{ prueba t para la igualdad de medias } \\
\hline & \multirow[b]{2}{*}{$\mathrm{F}$} & \multirow[b]{2}{*}{ Sig. } & \multirow[b]{2}{*}{$t$} & \multirow[b]{2}{*}{ gl } & \multirow{2}{*}{$\begin{array}{c}\text { Sig. } \\
\text { (bilat.) }\end{array}$} & \multirow{2}{*}{$\begin{array}{l}\text { Difer. de } \\
\text { medias }\end{array}$} & \multirow{2}{*}{$\begin{array}{c}\text { Difer. } \\
\text { error } \\
\text { están } \\
\text { d. }\end{array}$} & \multicolumn{2}{|c|}{$\begin{array}{c}95 \% \text { de interv. } \\
\text { de confianza } \\
\text { diferen. }\end{array}$} \\
\hline & & & & & & & & Inferior & Super. \\
\hline $\begin{array}{l}\text { Se asumen } \\
\text { varianzas iguales } \\
\text { No se asumen } \\
\text { varianzas iguales }\end{array}$ & 1,699 & 0,197 & $\begin{array}{l}-0,803 \\
-0,728\end{array}$ & $\begin{array}{r}73 \\
32,383\end{array}$ & $\begin{array}{l}\mathbf{0 , 4 2 4} \\
0,472\end{array}$ & $\begin{array}{l}-0,8012 \\
-0,8012\end{array}$ & $\begin{array}{l}0,9975 \\
1,1001\end{array}$ & $\begin{array}{l}-1,1868 \\
-1,4385\end{array}$ & $\begin{array}{l}2,7892 \\
3,0409\end{array}$ \\
\hline
\end{tabular}

Nota: La equivalente no paramétrica U de Mann-Whitney arroja un resultado similar (sig. $0,516)$.

De la misma forma, en la Tabla 8 comprobamos que la diferencia de algo más de un crédito en el total de financiera entre las universidades que no ofertan un grado de finanzas y contabilidad y las que sí que lo hacen, no se considera estadísticamente significativa asumiendo varianzas iguales según la prueba de Levene.

Tabla 8. Prueba de muestras independientes créditos TOTAL OBLIG. según GFYC Sí/NO

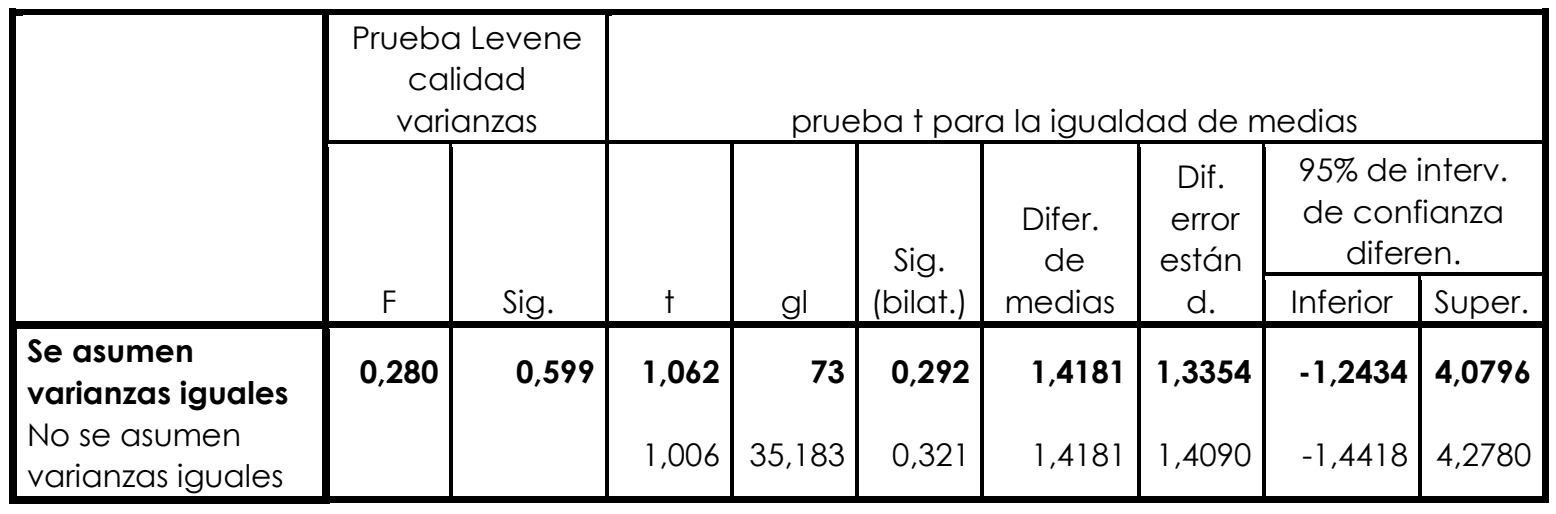
$0,408)$.

Nota: La equivalente no paramétrica U de Mann-Whitney arroja un resultado similar (sig.

Tratando de ver la dispersión de créditos obligatorios de contabilidad financiera por universidades y representando únicamente aquellas que destacan por estar claramente por debajo de la media, en contraposición con las que están claramente por encima de la media ( \pm 3 créditos) podemos ver la Figura 2. Se observa como destacan negativamente para la contabilidad las universidades Carlos III, Autónoma de Barcelona, Valencia, Oviedo y La Laguna de las públicas y la IE Universidad, San Jorge e Internacional Isabel I de Castilla de las privadas con tan sólo 6 créditos obligatorios de contabilidad financiera.

En el lado positivo para la contabilidad destacan muy por encima de la media las universidades públicas de Sevilla, Almería, Granada, Pablo Olavide, Las Palmas, León, la UNED, Alicante, Extremadura, La Coruña, Baleares, La Rioja, Miguel Hernández, Zaragoza, Cantabria, País Vasco y, sobre todo, Murcia y Santiago de Compostela. De las privadas destacan la Europea de Madrid, Navarra y la Universidad a distancia de Madrid (UDIMA). El triple de créditos de contabilidad financiera de todas estas universidades con respecto a las mencionadas en el bloque anterior parece una 
diferencia más que considerable a la hora de afrontar en condiciones óptimas las asignaturas optativas o un postgrado de especialización de contabilidad y auditoría sobre una base sólida.

Figura 2. Créditos obligatorios de contabilidad financiera por universidades

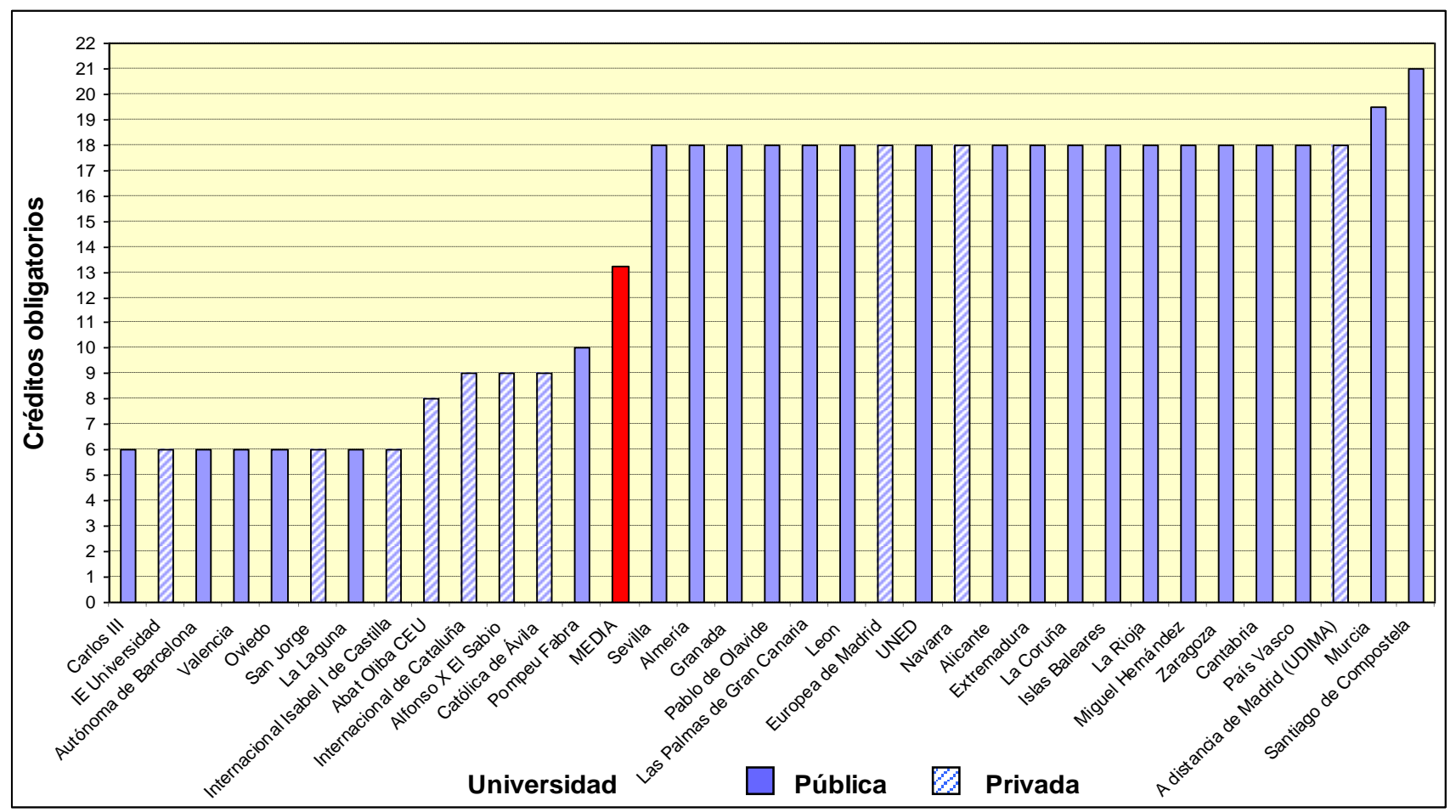

Si nos centramos en las universidades donde la contabilidad financiera sale poco favorecida para ver si tal vez compensan estas carencias con créditos de asignaturas relacionas con la contabilidad financiera (Figura 3), podemos observar que las únicas que compensan algo con una dedicación superior a la media $(5,96)$ son la de San Jorge con más de 8 créditos y las de La Laguna e Internacional Isabel I de Castilla con 12.

Figura 3. Créditos obligatorios relacionados con la contabilidad financiera

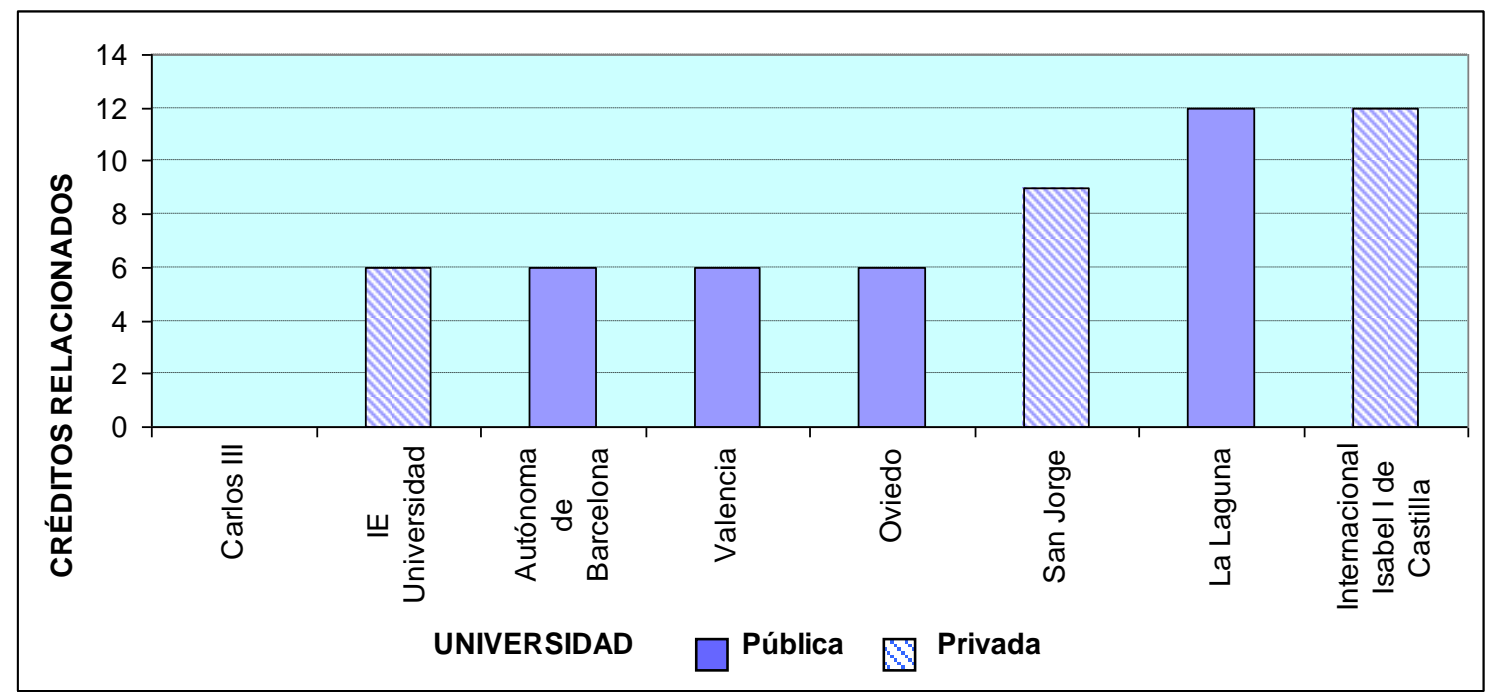


Por lo tanto, podemos concluir que las universidades públicas donde la contabilidad financiera está peor considerada son la Carlos III, la Autónoma de Barcelona, la de Valencia y la de Oviedo, mientras que de las privadas destaca la IE Universidad. En estas universidades los créditos dedicados a la contabilidad financiera suponen en el mejor de los casos $12(6+6)$, lo que significa un $6,34 \%$ de los créditos obligatorios que según el estudio de Cavero y Reig (2013) están entorno a 189. Si como aproximación de los créditos totales de contabilidad le sumamos la media de los créditos de costes que para las 13 universidades con menos contabilidad financiera se sitúa en 5,77 créditos, los créditos totales estarían en 17,77, es decir, un 9,40\% de la obligatoriedad y, por lo tanto, muy por debajo de los porcentajes recomendados en el libro blanco.

Factores que se atribuyen como favorables para conseguir un mayor número de créditos en contabilidad según el estudio de Cavero y Reig (2013, p. 70), como disponer de un departamento de Contabilidad independiente o contar con una mayor tradición y antigüedad de la docencia de contabilidad por venir de las anteriores Licenciaturas en Ciencias Empresariales no han funcionado en el caso de la Universidad de Oviedo ni en una de las que podríamos considerar como históricas, no ya como universidad sino como precursora de las Facultades de Economía como es la Universidad de Valencia. Sí que se confirma lo establecido por estos autores en cuanto a la gran variedad de créditos en contabilidad según los planes de estudio, no habiéndose seguido un criterio común, por lo que el grado de divergencia con respecto a las recomendaciones del Libro Blanco es considerable.

\section{LA OPTATIVIDAD EN CONTABILIDAD FINANCIERA}

Una amplia oferta de asignaturas optativas derivadas de la contabilidad financiera no debería ser la solución que contrarreste una carencia manifiesta de obligatoriedad de esta materia. Si la base de contabilidad financiera falla, asignaturas más avanzadas de contabilidad o más específicas están destinadas a fracasar. Fracasarán porque el nivel de exigencia tendrá que reducirse hasta el punto de que no suponga un obstáculo para que los alumnos opten por estas asignaturas 0 , simplemente, porque no tendrán demanda y la universidad acabará por reducir los grupos 0 , simplemente, no ofertarlas.

Y si un estudiante del grado de ADE ha tenido pocas asignaturas de contabilidad financiera, tendrá que renunciar a una gran cantidad de puestos de trabajo 0 competir en malas condiciones. En este sentido, para ser auditor, pueden tener problemas al solicitar al Instituto de Contabilidad y Auditoría de Cuentas la dispensa específica, relativa tanto al curso de formación teórica como a la primera fase del examen de determinadas materias, por no haber cursado materias equivalentes en contenido y duración.

Otra cuestión que también queremos destacar de la optatividad es el poco protagonismo que tiene la contabilidad informatizada a pesar de la importancia que se da en la actualidad a todo lo relacionado con las tecnologías de la información y comunicación (TICS). De las 75 universidades, tan sólo 4 ofertan de forma explícita una asignatura de este tipo. Habría que ver en cada caso si las prácticas, al menos, se hacen utilizando una aplicación informática. En este aspecto debemos tener también en cuenta que además del porcentaje que se le asigna específicamente a la contabilidad en los planes de estudio, una parte del porcentaje asignado a la informática también podría estar participado por la contabilidad informatizada, interactuando con cuestiones fiscales, de finanzas, de facturación y de control de almacenes. 
Finalmente queremos aportar como dato informativo que la media de créditos optativos de contabilidad financiera para el conjunto de universidades es de 9,32, destacando que hay 22 universidades sin optatividad ( 9 públicas de las cuales 5 son andaluzas y 13 privadas), siendo donde más créditos se ofertan en la de Girona (36), la Pompeu Fabra (30), el País Vasco (30), la de Valladolid (30), la Autónoma de Madrid (24), la Autónoma de Barcelona (24), la de Alcalá (24) y la de Valencia $(22,5)$. No olvidemos que, tal y como hemos puesto de manifiesto, la Autónoma de Barcelona y la de Valencia están entre las de menos base de contabilidad financiera, por lo que tratarían de recuperar el terreno perdido con una mayor oferta de optatividad.

\section{CONCLUSIONES}

Tradicionalmente se ha establecido como clave para mejorar la empleabilidad de los titulados universitarios que se dé una mayor coordinación entre lo que necesitan los empleadores y lo que ofrece la Universidad y, en este sentido, los actuales grados se conciben con un claro objetivo de cualificación profesional.

El Grado de Administración y Dirección de Empresas (ADE) se presenta como el de más éxito en la oferta del sistema universitario español, lo que se debe fundamentalmente a la gran demanda de los profesionales formados en las disciplinas propias de este grado. Y una de esas disciplinas es la contabilidad financiera, básica para interpretar la información económica del mundo de los negocios y poder tomar decisiones de forma razonada. Sin embargo, la discrecionalidad a la hora de confeccionar los planes de estudios en los distintos grados de las universidades españolas, hace que podamos encontrar diferencias muy importantes en cuanto a los créditos dedicados a la contabilidad financiera, lo que se colige con diferencias notables en otras materias.

Según el Libro Blanco de Economía y Empresa, la oferta de créditos en contabilidad para los grados de ADE debería estar entorno al $15 \%$ de los de formación básica y obligatoria, lo que ya supone de por sí una merma con respecto a los porcentajes que representaba en las antiguas Licenciaturas de Ciencias Empresariales y de ADE. Y tenemos que tener en cuenta que gran parte del éxito de los actuales grados de ADE se debe al trabajo desempeñado durante varias décadas por las anteriores licenciaturas, donde la contabilidad era una pieza clave. Por lo tanto, reducir la presencia de sus materias muy por debajo de ese porcentaje, tal y como ocurre en algunas universidades como la Carlos III, la IE Universidad, la Autónoma de Barcelona, la de Valencia o la de Oviedo, va en contra de lo considerado como normal por la trayectoria de estos estudios, por lo establecido en la mayoría de las universidades y por lo que demanda el mercado laboral para estos titulados. Debemos destacar al respecto que en el $80 \%$ de los grados de ADE de las universidades españolas, la contabilidad financiera tiene asignados al menos 12 créditos.

Y tampoco es normal que en un "hipotético contexto de convergencia con Europa" haya un gran número de universidades donde los créditos de contabilidad financiera tripliquen a las citadas en el párrafo anterior.

Tratando de valorar estadísticamente la diferencia de créditos de contabilidad financiera entre las universidades públicas y privadas podemos establecer que en éstas últimas, la media es significativamente menor. Si este mismo análisis lo empleamos para ver la influencia de ofertar paralelamente al grado de ADE el de Finanzas y Contabilidad, podemos establecer que esta circunstancia incide negativamente en los créditos pero no de forma significativa.

Si partimos de la base de que los créditos dedicados a las materias de contabilidad se redujeron al pasar de la Licenciatura de Ciencias Empresariales a la de ADE y también 
al pasar posteriormente a los grados y viendo lo que ha sucedido en algunas universidades con los créditos de contabilidad, la última reforma de los estudios superiores ha supuesto que los créditos atribuidos a esta materia no se correspondan con los que tradicionalmente han tenido y tienen en la mayoría de los grados de ADE. Así, nos gustaría que este trabajo sirva para aportar datos objetivos que permitan recuperar en futuras negociaciones o reformas el terreno perdido por la contabilidad en estas universidades.

La contabilidad financiera es la base sobre la que se sustentan diferentes materias consustanciales a los estudios de ADE (análisis de estados contables, auditoría, consolidación, contabilidades especiales, fiscalidad, etc.), además de ser parte activa en el lenguaje del mundo de los negocios. En este sentido, si en unos estudios claramente ligados a la gestión empresarial y económica, no se afronta con unos créditos mínimos y razonables de contabilidad, es fácil que se manifiesten una serie de carencias fundamentales, cuyas consecuencias pueden ser la falta de interés por las materias optativas relacionadas, un bajo nivel para afrontar un master de especialización en contabilidad a la vez que una difícil coexistencia con los estudiantes provenientes del grado de Finanzas y Contabilidad en su caso, una clara debilidad en materias que necesitan unos adecuados conocimientos de contabilidad, como por ejemplo las de tributación, y lo más grave de todo para un recién titulado, la insuficiente preparación para optar a muchos de los trabajos demandados por el mercado laboral para los que se supone que te has estado formando.

Finalmente, si tenemos en cuenta que en los últimos tiempos la investigación en contabilidad tampoco está apostando por la práctica profesional ni por la docencia, tal vez deberíamos reflexionar sobre lo que está ocurriendo y reconsiderar el papel que los profesores universitarios de contabilidad debemos desempeñar en la universidad y en la sociedad en general, planteándonos si verdaderamente realizamos la labor que se espera de nosotros.

Como futuras líneas de investigación se podría valorar y contrastar si los rankings que clasifican las universidades y los grados, atendiendo normalmente a su producción científica (investigación), coinciden con los que se elaboran a partir de la tasa de éxito de sus egresados a la hora de obtener un puesto de trabajo y de la percepción que tienen en las empresas sobre la formación adquirida en la universidad. De forma paradójica, en muchas de estas universidades donde el peso académico del Departamento de Contabilidad ha caído considerablemente tras la última reforma, su valoración y prestigio ha crecido considerablemente al medir su producción científica según las publicaciones en revistas indexadas básicamente como JCR, que tal y como se establece en el trabajo de Arquero et al. (2016), son los que menos utilidad tienen para la docencia de grado o la práctica profesional y está suponiendo el abandono de las cuestiones docentes y profesionales relevantes, e incluso de las líneas de investigación netamente contables. Así, los profesores que quieren hacer méritos para progresar laboralmente están más volcados en la investigación que en las cuestiones docentes y su importancia para la actividad profesional de los futuros egresados. En este sentido McGuigan (2015) y Sangster (2013 y 2015) destacan que la aplicación de sistemas basados únicamente en índices provocan una orientación cortoplacista, limitan severamente la libertad académica y la posibilidad de que los profesores obtengan evaluaciones justas con las consiguientes consecuencias académicas y psicosociales.

El actual sistema de avaluación de méritos basado en los JCRs hace que la investigación se vuelque en la realización de artículos internacionales publicados en áreas adyacentes (finanzas, economía de la empresa, economía, etc.) y se abandonen los trabajos en revistas profesionales y libros (Arquero et. al., 2017), ya que en España y en contabilidad escasean las revistas indexadas en este listado y las 
pocas que hay tienen índices de impacto bastante bajos (Carmona, 2006; Moizer, 2009; Argilés y García-Blandon, 2011). Tal y como ya advirtió Singleton-Green (2010), en las condiciones actuales, es posible tener una carrera relativamente exitosa en investigación en contabilidad sin llegar a tener una comprensión profunda de determinados aspectos de la materia.

Esta circunstancia plantea un debate en relación con el impacto que los sistemas de evaluación de la investigación puede llegar a tener sobre otras actividades académicas, como la docencia, la gestión y sobre la relación de los académicos con la práctica profesional (Arquero et. al., 2017). Y tal vez por ello, en los procesos de negociación de los planes de estudio se puede estar perdiendo la perspectiva de cuáles son los conocimientos de contabilidad con los que debe contar un futuro egresado para afrontar con éxito su incorporación al mercado laboral o, simplemente, que ante la dificultad para obtener publicaciones de impacto en contabilidad, los profesores de estas materias tengan cada vez menos peso en las decisiones que se toman (hay menos catedráticos a medida que hay menos acreditados, al menos en términos comparativos con otras áreas) y eso hace que paulatinamente se vea el área como secundaria.

\section{BIBLIOGRAFÍA}

ADECCO (2016): Informe Infoempleo Adecco: Oferta y demanda de empleo en España 2015. Madrid. Disponible en http://www.infoempleo.com/informeinfoempleo-adecco/.

ARGILÉS, J. M. Y GARCÍA-BLANDON J. (2011). Accounting research: A critical wiew of the present situation and prospects. Revista de Contabilidad, vol. 14, núm. 2: 9-34.

ARQUERO, J. L. Y DONOSO J. A. (2013). Docencia, investigación y burnout: el síndrome del quemado en profesores universitarios de Contabilidad. Revista de Contabilidad, vol. 16, núm. 2: 94-105.

ARQUERO, J. L., JIMÉNEZ, S. M. Y LAFFARGA, J. (2016): Utilidad percibida de la producción académica-contable. Opinión de los profesores universitarios y de los profesionales, Revista de Contabilidad - Spanish Accounting Review, vol. 19, núm. 2: 239-251. http://dx.doi.org/10.1016/j.rcsar.2015.10.004

ARQUERO, J. L., JIMÉNEZ, S. M. Y LAFFARGA, J. (2017): Patrones de investigación en contabilidad de los profesores con éxito académico, Revista Española de Financiación y Contabilidad - Spanish Journal of Finance and Accounting, vol. 46, núm. 3: 327-368, DOI: 10.1080/02102412.2017.1287462

CARMONA, S. (2006). Performance reviews, the impact of accounting research, an the role of publication forms. Advances in Accounting, vol. 22: 241-267.

CAVERO, J. A. Y REIG, J. (2013): El peso de la Contabilidad en el Grado de Administración y Dirección de Empresas, Revista de Contabilidad - Spanish Accounting Review, vol. 16, núm. 1: 66-73.

CONFERENCIA ESPAÑOLA DE DECANOS DE ECONOMÍA Y EMPRESA, CONFEDE (2004): Libro blanco del título de Grado en Economía y Empresa. Madrid. Diponible en http://www.aneca.es/var/media/150292/libroblanco_economia_def.pdf

CONFERENCIA ESPAÑOLA DE ORGANIZACIONES EMPRESARIALES, CEOE (2017): Libro blanco de los empresarios españoles. La educación importa. Madrid. Diponible en http://contenidos.ceoe.es/CEOE/var/pool/pdf/publications_docs-file-373-laeducacion-importa-libro-blanco-de-los-empresarios-espanoles.pdf 
DECLARACIÓN DE BOLONIA (1999): The European Higher Education Area. Joint declaration or harmonisation of the European Ministers of Education, Bolonia.

ESCOBAR, B., GARCÍA, E. Y LARRÁN, M. (2014): Factores que influyen sobre la producción científica en contabilidad en España: la opinión de los profesores universitarios de contabilidad (II parte), Revista Española de Documentación Científica, vol. 37, núm. 2, e047, DOl: 10.3989/redc.2014.2.1087

ESCOBAR, B. Y JIMÉNEZ, S. (2009). La implantación del grado de Finanzas y Contabilidad en el contexto del Espacio Europeo de Educación Superior: el caso de la Universidad de Sevilla, Revista Española de Financiación y Contabilidad, vol. XXXVIII, núm. 142: 293-310.

FEDERATION OF EUROPEAN ACCOUNTANTS, FEE (2015). The accountancy profession and taxation: doing the right thing, Tax Policy, October. Disponible en http://www.fee.be/images/publications/Tax_policy/1510_The_accountancy_professi on_and_taxation.pdf

FUNDACIÓN EVERIS (2017). III Ranking Universidad-Empresa: encuesta a las empresas españolas sobre la empleabilidad de los recién titulado, Madrid. Disponible en http://www.fundacioneveris.es

LEY ORGÁNICA 4/2007, de 12 de abril, por la que se modifica la Ley Orgánica 6/2001, de 21 de diciembre, de Universidades.

MCGUIGAN, N. (2015). The impact of journal rankings on Australasian accounting education scholarship-A personal view. Accounting Education, vol. 24, núm. 3: 187207.

MINISTERIO DE EDUCACIÓN, CULTURA Y DEPORTE (2003). La integración del sistema universitario español en el Espacio Europeo de Educación Superior, DocumentoMarco. Disponible en http://www.eees.es/pdf/Documento-Marco_10_Febrero.pdf

MOIZER, P. (2009). Publishing in accounting journals: A fair game? Accounting, Organization and Society, vol. 34, núm. 2: 285-304.

PARQUER, L. D., GUTHRIE, J. Y LINACRE, S. (2011). The relationship between academic accounting research and professional practice. Accounting, Auditing \& Accountability Journal, vol. 24, núm. 1: 5-14.

REAL DECRETO 1393/2007, de 29 de octubre, por el que se establece la ordenación de las enseñanzas universitarias oficiales.

SANGSTER, A. (2013). The effect of indexes, rankings and impact factors in accounting research and in accounting education in particular. Educade, vol. 4: 1-4.

SANGSTER, A. (2015). You cannot judge a book by its cover: The problems with journal rankings. Accounting Education. An International Journal, vol. 24, núm. 3: 175-186.

SINGLETON-GREEN, B. (2010). The communication gap: Why does't accounting research make a greater contribution to debates on accounting policy? Accounting in Europe, vol. 7, núm. 2: 129-145, DOI: 10.1080/17449480.2010.511880 